\title{
Cancellation of Critical Points in 2D and 3D Morse and Morse-Smale Complexes
}

\author{
Lidija Čmić $^{1}$ and Leila De Floriani ${ }^{2}$ \\ ${ }^{1}$ FTN, University of Novi Sad (Serbia) \\ comic@uns.ns.ac.yu \\ ${ }^{2}$ University of Genova (Italy) and University of Maryland (USA) \\ deflo@disi.unige.it
}

\begin{abstract}
Morse theory studies the relationship between the topology of a manifold $M$ and the critical points of a scalar function $f$ defined on $M$. The Morse-Smale complex associated with $f$ induces a subdivision of $M$ into regions of uniform gradient flow, and represents the topology of $M$ in a compact way. Function $f$ can be simplified by cancelling its critical points in pairs, thus simplifying the topological representation of $M$, provided by the Morse-Smale complex. Here, we investigate the effect of the cancellation of critical points of $f$ in Morse-Smale complexes in two and three dimensions by showing how the change of connectivity of a Morse-Smale complex induced by a cancellation can be interpreted and understood in a more intuitive and straightforward way as a change of connectivity in the corresponding ascending and descending Morse complexes. We consider a discrete counterpart of the Morse-Smale complex, called a quasi-Morse complex, and we present a compact graph-based representation of such complex and of its associated discrete Morse complexes, showing also how such representation is affected by a cancellation.
\end{abstract}

\section{Introduction}

The Morse-Smale complex is a widely used topological representation which describes the subdivision of a manifold $M$ into meaningful parts, characterized by uniform flow of the gradient of a scalar function $f$, defined on $M$. In applications, large data sets are usually interpolated by a continuous function, and then topological features are extracted, which represent the initial data in a compact way. Morse-Smale complexes can be applied for segmenting the graph of a scalar field for terrain modeling in $2 \mathrm{D}$, and recently some algorithms have been developed for segmenting three-dimensional scalar fields through Morse-Smale complexes 1115. For the review of work in this area, see 4 and the references therein.

The Morse-Smale complex of a scalar function $f$, defined on a manifold $M$, is the intersection of two complexes, namely the ascending and the descending Morse complexes. Intuitively, the descending cell of a critical point $p$ describes the flow of the gradient of $f$ towards $p$, and (dually) the ascending cell of $p$ describes the flow away from $p$. Each cell of a Morse-Smale complex is obtained as the intersection of two cells, the descending cell of a critical point $p$, and the

D. Coeurjolly et al. (Eds.): DGCI 2008, LNCS 4992, pp. 117-128, 2008.

(C) Springer-Verlag Berlin Heidelberg 2008 
ascending cell of a critical point $q$, and it describes the flow of the gradient vector field of $f$ from $q$ towards $p$.

One of the major issues when computing a representation of a scalar field as a Morse, or a Morse-Smale complex is the over-segmentation, due to the presence of noise in the data sets. To this aim, simplification algorithms have been developed, in order to eliminate less significant features from the Morse-Smale complex. The simplification is achieved by applying an operation called cancellation of critical points. A cancellation transforms one Morse-Smale complex into another, with fewer number of vertices, while maintaining the consistency of the underlying complex, and it enables the creation of a hierarchical representation. In 2D, the cancellation of a pair of critical points in a Morse-Smale complex consists of collapsing an extremum $p$ and a saddle $s$ into another extremum $q$ [189117]. Cancellation in 2D Morse complexes is considered in [3, where it is shown that cancellations reduce to well-known Euler operators [13]. Similar operations have been considered in the framework of $2 \mathrm{D}$ combinatorial maps [2].

Here, we investigate the effect of the cancellation in Morse-Smale complexes in two and three dimensions, and we show how the change of connectivity in a Morse-Smale complex, induced by a cancellation of pairs of critical points of $f$, can be interpreted and understood in a more intuitive and straightforward way as a change of connectivity in the corresponding ascending and descending Morse complexes. We then consider a discrete counterpart of the Morse-Smale complex, called a quasi-Morse complex, and we present a compact graph-based representation of such complex and of its associated discrete Morse complexes, showing also how such representation is affected by a cancellation. This representation, together with cancellation operations (and their inverse operations, called anticancellations) is a suitable basis for a multi-resolution representation of the topology of manifold $M$, induced by $f$.

The remainder of the paper is organized as follows. In Section 2, we recall some basic notions on cell complexes. In section [3, we review Morse theory, Morse and Morse-Smale complexes. In Section 4, we describe how a Morse-Smale complex is affected by cancellation of critical points of $f$, and we investigate how cancellation affects the structure of a Morse complex. In Section 5, we discuss the notion of a quasi-Morse complex, we introduce the incidence graph as a representation for encoding both the descending and ascending Morse complexes and the Morse-Smale complex, and we define modifications of incidence graph, induced by a cancellation. Concluding remarks are drawn in Section 6 .

\section{Background Notions}

We briefly review some basic notions on cell complexes. For more details on algebraic topology, see [14].

Intuitively, a Euclidean cell complex is a collection of basic elements, called cells, which cover a domain in the Euclidean space $\mathbb{R}^{n}[13$. A $k$-dimensional cell ( $k$-cell) $\gamma, 1 \leq k \leq n$, is a subset of $\mathbb{R}^{n}$ homeomorphic to an open $k$-dimensional ball $B^{k}=\left\{x \in \mathbb{R}^{k}:\|x\|<1\right\}$, with non-null (relative) boundary with respect 
to the topology induced by the usual topology of $\mathbb{R}^{n}$. A 0 -cell is a point in $\mathbb{R}^{n}$. The boundary of a 0 -cell is empty. $k$ is called the order or dimension of a $k$-cell $\gamma$. The boundary and closure of $\gamma$ are denoted $b d(\gamma)$ and $c l(\gamma)$, respectively.

A Euclidean cell complex is a finite set of cells $\Gamma$ in $\mathbb{R}^{n}$ of dimension at most $d, 0 \leq d \leq n$, such that $(i)$ the cells are pairwise disjoint, $(i i)$ for each cell $\gamma \in \Gamma$, the boundary $b d(\gamma)$ is the union of cells of $\Gamma$, (iii) if $\gamma, \gamma_{1} \in \Gamma$, such that $\operatorname{cl}(\gamma) \cap \operatorname{cl}\left(\gamma_{1}\right) \neq \emptyset$, then $\operatorname{cl}(\gamma) \cap \operatorname{cl}\left(\gamma_{1}\right)$ is the disjoint union of cells of $\Gamma$. The maximum $d$ of dimensions of cells $\gamma$ over all cells of a complex $\Gamma$ is called the dimension or the order of the complex. A subset $\Lambda$ of $\Gamma$ is called a subcomplex of $\Gamma$ if and only if $\Lambda$ is a cell complex. The $k$-skeleton of $\Gamma$ is the subcomplex of $\Gamma$, which consists of all cells of $\Gamma$ of dimension less than or equal to $k$. The domain (or carrier) $\Delta \Gamma$ of a Euclidean cell complex $\Gamma$ is the subset of $\mathbb{R}^{n}$ spanned by the cells of $\Gamma$. We shall consider two and three dimensional cell complexes $\Gamma$, such that $\Delta \Gamma$ is homeomorphic to a smooth compact two or three dimensional manifold $M$ without boundary. Recall that a $d$-manifold $M$ without boundary is a (separable Hausdoff) topological space in which each point $p$ has a neighborhood which is homeomorphic to $\mathbb{R}^{d}$.

An $h$-cell $\gamma^{\prime}$ which belongs to the boundary $b d(\gamma)$ of a cell $\gamma$ is called an $h$-face of $\gamma$. If $\gamma^{\prime} \neq \gamma$, then $\gamma^{\prime}$ is called a proper face of $\gamma$, and $\gamma$ and $\gamma^{\prime}$ are said to be incident. Two distinct $k$-cells $\gamma$ and $\gamma^{\prime}$ are adjacent if $(i)$ for $0<k \leq d$, there exists some $(k-1)$-cell of $\Gamma$ which is a face of both $\gamma$ and $\gamma^{\prime}$, and $(i i)$ for $0 \leq k<d$, there exists some $(k+1)$-cell of $\Gamma$ which contains both $\gamma$ and $\gamma^{\prime}$ as a face.

The space dual $\Gamma^{*}$ of a $d$-complex $\Gamma$ is a cell complex such that there is a one-to-one mapping from $\Gamma$ onto $\Gamma^{*}$ such that $(i)$ the image of a $k$-cell is a $(d-k)$-cell, $(i i)$ cells $\gamma$ and $\gamma^{\prime}$ are adjacent in $\Gamma$ if and only if their images are adjacent in $\Gamma^{*}$. The space dual $\Gamma^{*}$ of a complex $\Gamma$ is unique up to adjacency relation between cells.

\section{Morse Theory}

Morse theory studies relationships between the topology of a manifold, and (the critical points of) a function defined on a manifold. We review here the basic notions of Morse theory in the case of $d$-manifolds. For more details, see [16 15].

\subsection{Morse Functions}

Let $f$ be a $C^{2}$-differentiable real-valued function defined over a domain $D \subseteq \mathbb{R}^{d}$. A point $p \in \mathbb{R}^{d}$ is a critical point of $f$ if the gradient $\nabla f$ of $f$ vanishes on $p$, i.e., if $\nabla f(p)=0$. Function $f$ is said to be a Morse function if all its critical points are non-degenerate (the Hessian matrix Hess $f$ of the second derivatives of $f$ at $p$ is non-singular). Since all these properties are local, a Morse function $f$ can be defined on a $d$-dimensional manifold $M$. In some local coordinate system, there is a neighborhood of each critical point $p$ of $f$, in which

$$
f\left(x_{1}, x_{2}, \ldots, x_{d}\right)=f(p)-x_{1}-\ldots-x_{i}+x_{i+1}+\ldots+x_{d} .
$$


The number of minus signs in the above equality, i.e., the number of negative eigenvalues of $\mathrm{Hess}_{p} f$, is called the index of a critical point $p$. The corresponding eigenvectors show the directions in which $f$ is decreasing. A critical point $p$ is a minimum or a maximum if it has index 0 or $d$, respectively. Otherwise, if index of $p$ is $i, 0<i<d, p$ is an $i$-saddle.

An integral line $c(t)$ of a function $f$ is a maximal path which is everywhere tangent to $\nabla f$. Thus, $c(t)$ follows the direction in which the function has the maximum increasing growth. Each integral line connects two distinct critical points of $f$, called its origin and destination. In 2D, each saddle $s$ is incident to two ascending and two descending integral lines, which connect $s$ to two (not necessarily distinct) maxima and two (not necessarily distinct) minima, respectively. These ascending and descending integral lines alternate cyclically around $s$. In $3 \mathrm{D}$, each 1-saddle (2-saddle) $s$ is incident to two descending (ascending) integral lines, which connect $s$ to two (not necessarily distinct) minima (maxima).

An operation called cancellation of critical points is defined on the set of critical points of $f$. It eliminates the critical points of $f$ in pairs. If $p$ and $q$ are two critical points of $f$, such that $p$ is of index $i, q$ is of index $i+1$, and such that there is a unique integral line $c$ having $p$ as origin and $q$ as destination, then $f$ can be perturbed to another Morse function $g$ defined on $M$, such that critical points of $g$ coincide in position and in index with the remaining critical points of $f$ (except $p$ and $q$ ). A cancellation of a pair of critical points $p$ and $q$ modifies the gradient field along $c$, and in a small neighborhood of $c$, thus simulating the smoothing of $f$.

\subsection{Morse and Morse-Smale Complexes}

Let $f: M \rightarrow \mathbb{R}$ be a Morse function, where $M$ is a compact $d$-manifold without boundary. Integral lines that converge to (originate from) a critical point $p$ of index $i$ form an $i$-cell $((d-i)$-cell) called a stable (unstable) manifold (or descending (ascending) manifold) of $p$. The descending (ascending) manifolds are pairwise disjoint and they decompose $M$ into open cells which form a complex, since the boundary of every cell is a union of lower-dimensional cells. Such complexes are called descending and ascending Morse complexes.

If $p$ is a minimum, the descending manifold of $p$ is $p$ itself, while if $p$ is a 1-saddle, the descending manifold of $p$ is an open 1-cell bounded by two (not necessarily distinct) minima. If $p$ is a maximum in a $2 \mathrm{D}$ manifold $M$, the descending manifold of $p$ is an open 2-cell, bounded by a sequence of (at least one) minima and (at least one) descending 1-cells of saddles. In $3 \mathrm{D}$, the boundary $B$ of a descending three-cell of a maximum $p$ consists of (at least one) descending 2-cells of 2-saddles, and the boundaries of these 2-cells consist of descending 1-cells of 1-saddles on $B$, and (at least one) minima.

A Morse function $f$ is called a Morse-Smale function if and only if the descending and the ascending manifolds intersect transversally. This means that the intersection (if it exists) of the descending $i$-dimensional manifold of a critical point $p$ of index $i$, and the ascending $(d-j)$-dimensional manifold of a critical point $q$ of index $j, i \geq j$, is an $(i-j)$-dimensional manifold. Cells that are 
obtained as the intersection of descending and ascending manifolds of a MorseSmale function $f$ decompose $M$ into a Morse-Smale complex.

In 2D, each 2-cell of a Morse-Smale complex is related to a maximum $p$ and a minimum $q$, as it is obtained as the intersection of the descending 2-manifold of $p$ and the ascending 2-manifold of $q$. Such 2-cell is quadrangular, with vertices $q, s_{1}, p, s_{2}$ (of index $0,1,2,1$ ), in this order along the boundary. These 2-cells are obtained as union of two triangles, namely $q, s_{1}, p$ and $q, s_{2}, p$, where $s_{1}$ and $s_{2}$ are the (not necessarily distinct) saddles, which are in the boundary of both the descending 2-manifold of $p$ and the ascending 2-manifold of $q$. A maximum $p$ and a minimum $q$ may determine more than one 2-cell of a Morse-Smale complex.

In 3D, each 3-cell of a Morse-Smale complex is related to a maximum $p$ and a minimum $q$. Such 3-cell is obtained as union of tetrahedra of the form $q, s_{1}, s_{2}, p$, where $s_{1}$ and $s_{2}$ are 1-saddles and 2-saddles, which are in the boundary of both the descending 3-manifold of $p$ and the ascending 3-manifold of $q$.

If $f$ is a Morse-Smale function, then the ascending and the descending complex of $f$ are dual to each other. Each critical point $p$ of index $i$ of a Morse-Smale function $f$ corresponds to a vertex (which we denote by $p$ ) of a Morse-Smale complex of $f$, and it also corresponds to the descending $i$-dimensional manifold of $p$, i.e., to an $i$-cell of the descending Morse complex (denoted by $p$ ), and to the ascending $(d-i)$-dimensional manifold of $p$. We illustrate the correspondence between Morse and Morse-Smale complexes in Figure 1 .
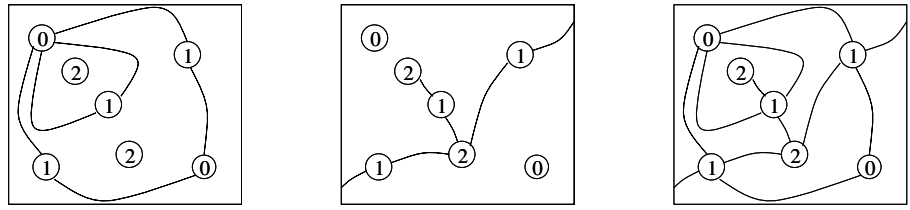

Fig. 1. A Morse-Smale function $f$, defined on a 2-dimensional domain. Numbers indicate the index of the critical points. (left) Descending Morse complex. (middle) Ascending Morse complex. (right) Morse-Smale complex.

\section{Cancellations in Morse-Smale and Morse Complexes}

Although Morse and Morse-Smale complexes describe the topology of a manifold $M$ in a more compact way than the full dataset, it is often desirable to reduce the number of critical points of $f$, thus obtaining a simplified representation of the topology of $M$. This can be achieved by applying the operation of cancellation of (pairs of) critical points. A pair of critical points $p$ and $q$ of function $f$ can be cancelled if $p$ and $q$ have consecutive indices $i$ and $i+1$, respectively, and there is a unique integral line connecting them. In the Morse-Smale complex of $f$, vertices $p$ and $q$ are adjacent and connected through a single edge, while in the Morse complex, cell $p$ is in the boundary of cell $q$, and $p$ appears only once 
in the boundary of $q$. Specifically, a vertex $p$ and an edge $q$ may be cancelled if $q$ is not a loop with endpoint $p$. An edge $p$ and a face $q$ may be cancelled if in a cyclic order of edges in the boundary of $q$, edge $p$ appears only once. In $2 \mathrm{D}$, this means that the unique other face $r$ incident to $p$ is different from $q$. Finally, (in 3D) a face $p$ and a 3-cell (volume) $q$ may be cancelled if the unique other 3 -cell $r$ incident to $p$ is different from $q$.
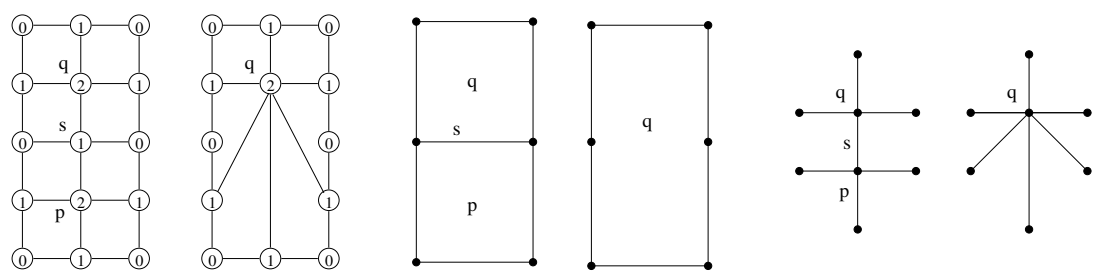

Fig. 2. Cancellation of a maximum $p$ and a saddle $s$ in a 2D (left) Morse-Smale complex, (middle) descending Morse complex (edge removal), (right) ascending complex, and dually, cancellation of a minimum $p$ and a saddle $s$ in the descending complex (edge contraction). Numbers indicate the index of the corresponding critical point.

In 2D, a saddle and an extremum are cancelled. Cancellation of a maximum $p$ and a saddle $s$ in a Morse-Smale complex is illustrated in Figure 2(left). It can be viewed as merging $p$ and $s$ into the other maximum $q$ adjacent to saddle $s$, while at the same time all four edges incident in $s$ are deleted. If $q=p$, cancellation is forbidden. Note that we are only interested in the changes of connectivity in a Morse-Smale complex, while we do not take into account any considerations about the feasibility of a cancellation, which are induced by function values. In the example above, $f(q)$ has to be greater than the value of $f$ at any of the saddles which become connected with $q$, if the cancellation of $p$ and $s$ is to be allowed. In a descending Morse complex, cancellation corresponds to merging the 2-cells $p$ and $q$ into $q$, i.e., to the removal of edge $s$, as illustrated in Figure 2 (middle). In a Morse-Smale complex, each saddle which was previously connected to $p$ is connected to $q$ after cancellation. Correspondingly, in the descending Morse complex, each edge which was previously on the boundary of 2 -cell $p$ is on the boundary of 2-cell $q$ after cancellation. Similarly, the cancellation of a saddle and a minimum corresponds to an edge contraction, which is the dual operation of edge removal, as illustrated in Figure 2, The reverse is true for the ascending complex. The order in which the pairs of points are canceled can be determined based on the notion of persistence 91.

Simplification of 3D Morse-Smale complexes has been investigated only recently [10]. In 3D there are three possible types of cancellations: minimum and 1-saddle, 1-saddle and 2-saddle, and 2-saddle and maximum. While the two cancellations involving a minimum or a maximum are similar to the ones performed in the $2 \mathrm{D}$ case, the saddle-saddle cancellation does not have an analog in $2 \mathrm{D}$. 
A cancellation of a minimum and a 1-saddle, or of a maximum and a 2saddle, in a Morse-Smale complex, can be interpreted as merging the two canceled points, an extremum point $p$ and a saddle $s$ into the unique extremum point $q$ which is the other endpoint of the (descending or ascending) 1-manifold of saddle $s$. As in the 2D case, if $q=p$, cancellation is forbidden. Edges and faces which were incident in $s$ are deleted from the Morse-Smale complex, and $q$ will replace $p$ in all edges and faces which had $p$ as one of their vertices. In a 3D descending Morse complex, cancellation of a 1-saddle and a minimum corresponds to an edge contraction, while cancellation of a maximum and a 2-saddle corresponds to the removal of a face. The modifications of (a part of) a Morse-Smale and of the descending and ascending Morse complexes, induced by a cancellation of a maximum $p$ and a 2-saddle $s$, are illustrated in Figure 3 .
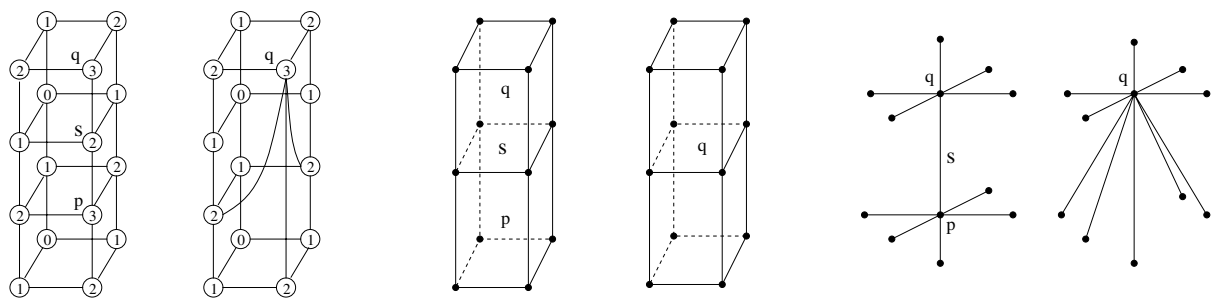

Fig. 3. Cancellation of a maximum $p$ and a 2-saddle $s$ in 3D in a (left) Morse-Smale complex, (middle) descending Morse complex (face removal), (right) ascending Morse complex, and dually, cancellation of a minimum $p$ and a 1-saddle $s$ in the descending complex (edge contraction). Numbers indicate the index of the corresponding critical point.

A cancellation of a 1-saddle $p$ and a 2-saddle $q$ makes the extension from $2 \mathrm{D}$ to $3 \mathrm{D}$ non-trivial. When $p$ and $q$ are cancelled, the descending and ascending manifolds of $p$ and $q$ are deleted from the Morse-Smale complex, and the remaining points on the boundary of these 2-manifolds are reconnected. Each 2-saddle, which was adjacent to $p$, becomes adjacent to each 1-saddle, which was adjacent to $q$, as illustrated in Figure 4 (left). In this way, the number of cells in the Morse-Smale complex increases, although the number of critical points (vertices of the Morse-Smale complex) decreases by two. The cancellation of a 2 -saddle $q$ and a 1-saddle $p$ in a descending Morse complex is illustrated in Figure 4 (right). Edge $p$ and face $q$ are deleted, and the boundary of each face incident in $p$ is extended to include the boundary of face $q$ (with the exception of edge $p$ ). It can be viewed as each face incident in $p$ is extended to include a copy of face $q$, keeping an infinitesimal separation between these copies. Each edge which was on the boundary of face $q$ is, after cancellation, on the boundary of all other faces incident to $p$. Thus, all the vertices (minima) on the boundary of face $q$ will be, after cancellation, on the boundary of all faces incident to edge $p$, and on the boundary of all volumes (maxima) incident to $p$. All such minima-maxima pairs induce new cells in the Morse-Smale complex, although the number of cells in the Morse complexes decreases by two. 

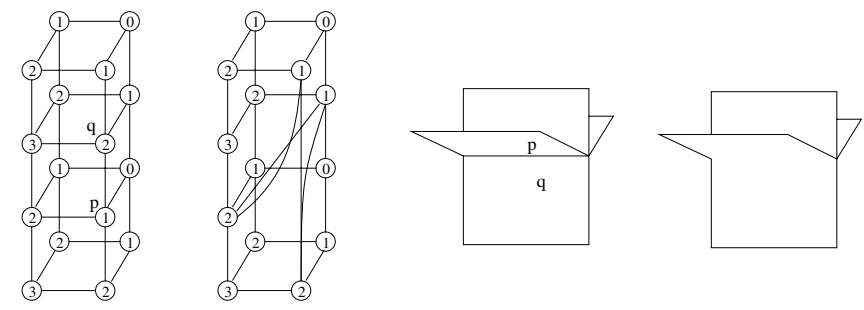

Fig. 4. Cancellation of a 1-saddle $p$ and a 2-saddle $q$ in 3D in a (left) Morse-Smale complex, (right) descending Morse complex. Numbers indicate the index of the corresponding critical point.

\section{Morse-Smale Complexes in the Discrete Case}

In this Section, we recall the notion of a quasi-Morse complex, and we introduce the incidence graph as a compact, dual representation of descending and ascending Morse complexes.

\subsection{Quasi-Morse Complexes}

In order to capture the combinatorial structure of a Morse-Smale complex of a manifold $M$, without making reference to a function $f$, a notion of a quasi-Morse complex in 2D and 3D is introduced in [9] and 8, respectively.

In 2D, a cell complex $\Gamma$ is a quasi-Morse complex if it is a subdivision of $M$, in which the set of vertices (0-cells) of $\Gamma$ is partitioned into three sets $U, V$ and $W$ and the set of edges (1-cells) of $\Gamma$ is partitioned into two sets $A$ and $B$ with the following properties: $(i)$ there is no edge which connects two vertices from $U \cup W$, or two vertices from $V$, (ii) edges in $A$ have endpoints in $U \cup V$ and edges in $B$ have endpoints in $V \cup W$, (iii) each vertex $p \in V$ belongs to four edges, which alternate between $A$ and $B$ in a cyclic order around $p,(i v)$ all 2-cells of $\Gamma$ are quadrangles, with vertices from $U, V, W, V$, in this order along the boundary.

In 3D, a cell complex $\Gamma$ is a quasi-Morse complex if it is a subdivision of $M$, in which the set of vertices of $\Gamma$ is partitioned into four sets $U, V, X$ and $Y$, the set of edges of $\Gamma$ is partitioned into three sets $R, S$, and $T$ and the set of 2-cells of $\Gamma$ is partitioned into two sets $P$ and $Q$ with the following properties: ( $i$ ) edges from $R, S$, and $T$ connect vertices from $U$ and $V, V$ and $X$, and $X$ and $Y$, respectively, and 2-cells from $P$ and $Q$ are quadrangles with nodes from $U, V, X, V$, and $V, X, Y, X$, in that order, respectively, along the boundary, (ii) there are no vertices within 1-, 2-, and 3-cells of $\Gamma$, (iii) each edge in $S$ is on the boundary of four quadrangles, which in a cyclic order alternate between $P$ and $Q$. In 3D, a quasi-Morse complex is more restrictive than a Morse-Smale complex. In the Morse complexes corresponding to a quasi-Morse complex, faces bounded by only one vertex, and edges which do not bound a face, are not allowed, since they would imply the existence of faces in a quasi-Morse complex, which are not quadrangles. 
Thus, in a quasi-Morse complex, the cancellation of an edge $e$ and a face $f$ is forbiden if $(i) e$ is the only edge in the boundary of $f$, and there is a face different from $f$, bounded only by $e$, or $(i i) e$ is bounding only $f$, and there is an edge different from $e$, which is bounding only $f$. Otherwise, a cancellation could result in a face bounded by only one vertex, and an edge not bounding a face, respectively. Cancellation of a minimum (vertex) $p$ and a 1-saddle (edge) $q$ is allowed if there is no 2 -saddle (face) $r$ such that $q$ is the only edge in the boundary of $r$. Dually, cancellation of a maximum (volume) $p$ and a 2-saddle (face) $q$ is allowed if there is no 1-saddle (edge) $r$ such that $r$ bounds no other face but $q$. These situations are illustrated in Figure 5

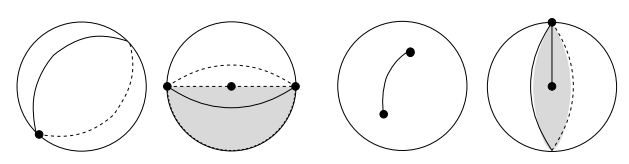

Fig. 5. (left) Forbiden face-edge cancellation. (right) Forbiden vertex-edge and volumeface cancellation.

\subsection{Dual Representation of Morse Complexes}

We encode the descending Morse complex $\Gamma_{d}$, obtained from a quasi-Morse complex of a Morse-Smale function, using an incidence graph $I G=(C, A)$.

The Incidence Graph (IG) [7] is an incidence-based explicit data structure for cell complexes in arbitrary dimensions. The topological information captured by the $I G$ is the set of incidence relations among cells that differ by exactly one dimension. Formally, the $I G$ encodes all the cells of any given $d$-dimensional cell complex $\Gamma$, and for each $i$-cell $\gamma$, its immediate boundary, and immediate co-boundary relations, namely all $(i-1)$-cells in the boundary of $\gamma$, and all $(i+1)$-cells in the co-boundary of $\gamma$. An incidence graph corresponds to the Hasse diagram describing the complex [12. If we turn the incidence graph $I G$ of a descending complex $\Gamma_{d}$ 'upside-down', we obtain the incidence graph of the ascending complex $\Gamma_{a}$, which is dual to $\Gamma_{d}$. Thus, the incidence graph encodes simultaneously both the ascending and the descending Morse complexes. In Figure 6 (left), a cell complex describing the subdivision of the extended plane, its dual complex, and the corresponding incidence graph are illustrated.

An incidence graph $I G$ encodes directly the 1-skeleton of a quasi-Morse complex, since the nodes of the $I G$ correspond to the critical points of $f$, and there is an arc in the $I G$ connecting two nodes $p$ and $q$ if and only if $p$ and $q$ are connected by an edge in the quasi-Morse complex. Other cells of a Morse-Smale complex are encoded implicitly. In 2D, all 2-cells of a Morse-Smale complex, associated with a maximum $p$ and a minimum $q$, are obtained as the union of two triangles $p, s_{1}, q$ and $p, s_{2}, q$, where $s_{1}$ and $s_{2}$ are two (not necessarily distinct) saddles which are connected to both $p$ and $q$ in $I G$. In 3D, all 3-cells of a MorseSmale complex, associated with a maximum $p$ and a minimum $q$, are obtained as the union of tetrahedra of the form $q, s_{1}, s_{2}, p$, where $s_{1}$ are 1-saddles which 
are connected to $q, s_{2}$ are 2-saddles which are connected to $p$, and $s_{1}$ and $s_{2}$ are connected to each other in $I G$. 2-cells in a 3D Morse-Smale complex are associated either with a maximum $p$ and a 1-saddle $s_{1}$, or with a minimum $q$ and a 2 -saddle $s_{2}$, and are obtained in a similar way as the 2-cells in a 2D Morse-Smale complex. Note that an incidence graph $I G$ cannot encode a general Morse complex, since in $I G$ there is no possibility to encode, for example, a face bounded by just one vertex.

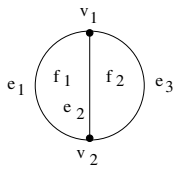

$\mathrm{f}_{3}$

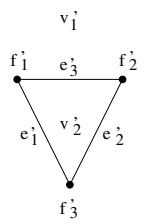

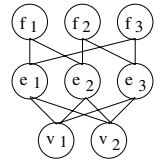

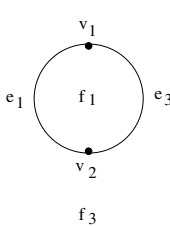

$\mathrm{f}_{3}$
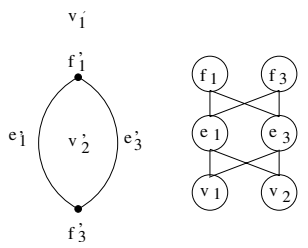

Fig. 6. (left) A 2-dimensional cell complex $\Gamma_{d}$, which consists of vertices $v_{1}$ and $v_{2}$, edges $e_{1}, e_{2}$ and $e_{3}$, and faces $f_{1} f_{2}$ and $f_{3}$, the dual complex $\Gamma_{a}$, and incidence graph $I G$ of $\Gamma_{d}$ and $\Gamma_{a}$. (right) $\Gamma_{d}, \Gamma_{a}$, and $I G$, after the cancellation of edge $e_{2}$ and face $f_{2}$.

Cancellation of critical points in a quasi-Morse complex can be formalized in terms of the incidence graph. Let $I G=(C, A)$ be the incidence graph of the quasi-Morse complex associated with $f$, and let $p$ and $q$ be two critical points of $f$ of consecutive index $i$ and $i+1$, respectively, such that there is a unique integral line of $f$ joining $p$ and $q$. Then $p$ and $q$ are nodes of the $I G$, which belong to two consecutive layers of the $I G$, such that $p$ and $q$ are connected by a unique arc of $I G$. Let us denote by $I^{+}$the set of arcs incident to either $p$ or $q$. We will call this set the influence set of the cancellation $u_{c}(p, q)$ of $p$ and $q$. For each $\operatorname{arc}(p, s) \in I^{+}$, where $s$ is an $(i+1)$-cell and $s \neq q$, we call $s$ a relevant $(i+1)$-cell, and similarly for each $\operatorname{arc}(q, r) \in I^{+}$, where $r$ is an $i$-cell and $r \neq p$, we call $r$ a relevant $i$-cell of update $u_{c}(p, q)$. Cancellation of $p$ and $q$ eliminates $p$ and $q$ from $I G$, together with arcs from $I^{+}$, and introduces new set of arcs, denoted $I^{-}$connecting all relevant $i$-cells $r$, to all relevant $(i+1)$-cells $s$. Thus, after the cancellation of $p$ and $q$, the incidence graph $I G=(C, A)$ of $f$ is transformed into another incidence graph $I G^{\prime}=\left(C^{\prime}, A^{\prime}\right)$, such that $C^{\prime}=$ $C \backslash\{p, q\}, A^{\prime}=\left(A \backslash I^{+}\right) \cup I^{-}$. Cancellation of edge $e_{2}$ and face $f_{2}$ in cell complex (and the corresponding incidence graph) from Figure 6 (left) is illustrated in Figure 6 (right).

Another condition needs to be imposed since $I G^{\prime}$ needs to have the structure of an incidence graph, as explained above. We formulate these conditions in terms of the incidence graph. Cancellation of a 1-cell $p$ and a 2-cell $q$ should be forbidden if $(i)$ there are no relevant 1-cells, and there is a 2-cell $s$ which is connected to no 1 -cell other than $p$, or $($ ii $)$ there are no relevant 2 -cells, and there is a 1-cell $r$ which is connected to no 2-cell other than $q$. Cancellation of a 0-cell $p$ and a 1-cell $q$ should be forbidden if there is a 2-cell $s$, which is connected to no 1-cell other than $q$, while cancellation of a 2-cell $p$ and a 3-cell $q$ should be forbidden if there is a 1-cell $r$ which is connected to no 2-cell other than $p$. 


\section{Concluding Remarks}

We have recalled the notion of cancellation of critical points of a Morse function $f$, and have shown how the changes of connectivity in a Morse-Smale complex of $f$ after a cancellation are easier to understand if interpreted as changes of connectivity in a Morse complex of $f$.

We have considered the quasi-Morse complex as the combinatorial structure of the Morse-Smale complex in the discrete case and we have discussed the incidence graph as a representation for quasi-Morse complexes. This representation encodes both the ascending and the descending complexes associated with a quasi-Morse complex. The incidence graph for a 3D scalar field can be constructed by applying the algorithm described in [5], which computes the two dual Morse complexes through an efficient discrete approach that does not involve any floating-point computation.

We are currently working on a definition of a multi-resolution topological model for 3D scalar fields, based on the cancellation operators discussed and on the notion of dependency of the modifications of a cell complex. This will lead to a morphology-based description of a 3D scalar field at different levels of abstraction, based on the framework on multi-resolution modeling introduced in 6]. Further developments of this work are concerned with extending the formalization proposed here to the $n$-dimensional case.

\section{Acknowledgements}

This work has been partially supported by the European Network of Excellence AIM@SHAPE under contract number 506766, by the National Science Foundation under grant CCF-0541032, by the MIUR-FIRB project SHALOM under contract number RBIN04HWR8 and by the MIUR-PRIN project on "Multiresolution modeling of scalar fields and digital shapes".

\section{References}

1. Bremer, P.-T., Edelsbrunner, H., Hamann, B., Pascucci, V.: A Topological Hierarchy for Functions on Triangulated Surfaces. Transactions on Visualization and Computer Graphics 10(4), 385-396 (2004)

2. Brun, L., Kropatsch, W.G.: Dual Contraction of Combinatorial Maps. Technical Report PRIP-TR-54, Institute for Computer Aided Automation 183/2, Pattern Recognition and Image Processing Group, TU Wien, Austria (1999)

3. Comić, L.: On Morse-Smale Complexes and Dual Subdivisions. In: 4th SerbianHungarian Joint Symposium on Intelligent Systems (SISY 2006), Subotica, Serbia, September 29-30, pp. 265-274 (2006)

4. Čomić, L., De Floriani, L., Papaleo, L.: Morse-Smale Decompositions for Modeling Terrain Knowledge. In: Cohn, A.G., Mark, D.M. (eds.) COSIT 2005. LNCS, vol. 3693, pp. 426-444. Springer, Heidelberg (2005) 
5. Danovaro, E., De Floriani, L., Mesmoudi, M.M.: Topological Analysis and Characterization of Discrete Scalar Fields. In: Asano, T., Klette, R., Ronse, C. (eds.) Geometry, Morphology, and Computational Imaging. LNCS, vol. 2616, pp. 386402. Springer, Heidelberg (2003)

6. De Floriani, L., Magillo, P., Puppo, E.: Multiresolution Representation of Shapes Based on Cell Complexes (invited paper). In: Bertrand, G., Couprie, M., Perroton, L. (eds.) DGCI 1999. LNCS, vol. 1568, pp. 3-18. Springer, Heidelberg (1999)

7. Edelsbrunner, H.: Algorithms in Combinatorial Geometry. Springer, Heidelberg (1987)

8. Edelsbrunner, H., Harer, J., Natarajan, V., Pascucci, V.: Morse-Smale Complexes for Piecewise Linear 3-Manifolds. In: Proceedings 19th ACM Symposium on Computational Geometry, pp. 361-370 (2003)

9. Edelsbrunner, H., Harer, J., Zomorodian, A.: Hierarchical Morse Complexes for Piecewise Linear 2-Manifolds. In: Proceedings 17th ACM Symposium on Computational Geometry, pp. 70-79 (2001)

10. Gyulassy, A., Natarajan, V., Pascucci, V., Bremer, P.-T., Hamann, B.: Topologybased Simplification for Feature Extraction from 3D Scalar Fields. In: Proceedings IEEE Visualization 2005, pp. 275-280. ACM Press, New York (2005)

11. Gyulassy, A., Natarajan, V., Pascucci, V., Hamann, B.: Efficient Computation of Morse-Smale Complexes for Three-Dimensional Scalar Functions. In: Proceedings IEEE Visualization 2007, Sacramento, California, October 28 - November 1, ACM Press, New York (2007)

12. Lewiner, T.: Geometric Discrete Morse Complexes. PhD thesis, Department of Mathematics, PUC-Rio, Advised by Hélio Lopes and Geovan Tavares (2005)

13. Mantyla, M.: An Introduction to Solid Modeling. Computer Science Press (1987)

14. Massey, W.S.: A Basic Course in Algebraic Topology. In: Graduate Texts in Mathematics, vol. 127. Springer, Heidelberg (1991)

15. Matsumoto, Y.: An Introduction to Morse Theory. Translations of Mathematical Monographs, vol. 208. American Mathematical Society (2002)

16. Milnor, J.: Morse Theory. Princeton University Press, New Jersey (1963)

17. Takahashi, S., Ikeda, T., Kunii, T.L., Ueda, M.: Algorithms for Extracting Correct Critical Points and Constructing Topological Graphs from Discrete Geographic Elevation Data. Computer Graphics Forum 14, 181-192 (1995)

18. Wolf, G.W.: Topographic Surfaces and Surface Networks. In: Rana, S. (ed.) Topological Data Structures for Surfaces, pp. 15-29. John Wiley, Chichester (2004) 